\title{
The Future of Science: Big Data Meets Scholarly Impact
}

by Tasneem Badshah, Tony Xu, Shayan Khalili, Cynthia Deng, Peter Chou, Kevin Hong, Chandler Lel, Haolin Zhang, Charlie Sun, Kerry Li, Zhenyu Li, Alex Bogdan, Yingning Gui, Henry Lin, Brennan Lu, Vic Li, Cecilia Shi, Michael Yang, Eva Zhang, Suzy Zhang, George Li, Michal Fishkin, Jennifer Ou, Andrew Zhu, Thomas Beckley, William Kwong, Danny Pechersky, Natalie Nova, Michael Pavia, Andrew Schmittat, Leon Chen, Curtis Chong, Emily Huang, Nathan Lo, Montgomery Gole, Sean Malins-Umansky, Patrick Prochazka, Tony Zhang, Joseph Train, David Roizenman, Seth Damiani, Ronny Rochwerg, Lunjun Zhang, Justin Palombo, Sarah Costa, Jamie Birker, Jesse Becker, Jessica Casalino, Anya Filipas, Akera Otto, Lily Chen, Jenny Chen, Valerie Hermanns, Daniel Grignano, Andrew Latobesi, Mark Ho.

STEM Fellowship's Big Data Challenge is a unique pedagogical experiment, providing an inquiry and learning experience for high school students that, upon equipping them with top-notch analytical tools, tasks them to find hidden patterns and trends in complex socioeconomic or scientific data. This year's challenge provided a multidisciplinary competitive opportunity; over a period of two and a half months, teams analyzed scholarly impact data through the prism of computational methods, all in order to answer the question: What is the future of science?

Published here are the abstracts from all entrants. While scholarly impact data - as graciously provided by Altmetric - was the overall focus of the competition, teams applied a wide variety of perspectives in their respective projects, approaching the data through various angles that included research funding, gender diversity, Twitter interactions, and more. The tools used by teams were similarly diverse, ranging from SAS Studio and Tableau to $R$ and Python.

For all the variation between project themes, it remains that all submissions are of incredibly high quality. Every paper is demonstrative of immense creativity and high potential on the respective team's part.

On behalf of STEM Fellowship, I would like to extend my heartfelt congratulations to all students who participated in the challenge, and I wish them all the best for their future endeavours in research and data science. It has been a privilege for us to witness the anaIytical capabilities of the next generation of students firsthand, and I am certain all entrants will only continue to demonstrate excellence in their respective research careers.

\section{Ahmed Hasan \\ Director for Big Data Education, STEM Fellowship \\ Big Data Challenge 2016-2017 Team Lead}

\section{Disclaimer}

These abstracts are provided for all student teams that have submitted project reports by January 15, 2017. The STEM Fellowship Journal editorial board has made every effort to ensure proof and English editing of these abstracts in a limited amount of time, and neither organization as a whole or any of its volunteer members can be held accountable for inaccuracies that may have occurred in the abstract publication. Abstracts are published in alphabetical orderper school names of the participating teams.

\section{Who Cares About Tweeting? The Impact of Social Media on the Future of Scientific Research \\ Tasneem Badshah \\ The Abelard School \\ (Abelard 1)}

\section{Abstract}

Social media has become explosively influential in our daily lives, and it has become increasingly important in scientific research. In this age of social media-driven interaction, researchers are constantly looking for ways to promote and spread their research. It is interesting to see how social media actually impacts research and Altmetric provides a way to understand this, especially in comparison to more traditional metrics. With the Altmetric data, we are able to track more modern indicators of engagement. We decided to look into the specific question of: Does the number of tweets about a research paper actually impact the number of readers on Mendeley? We used the diabetes dataset provided by Altmetric and looked into the correlation between the number of tweets and readers for each research paper. We also looked into the correlation for different subgroups of tweeters. We concluded that there was no linear correla- 
tion, and because the number of tweets does not strongly impact the number of reads, we cannot predict an increase in reads if an article is actively tweeted about. According to our analysis, there is no straightforward way of determining whether increased tweeting will have a strong positive contribution to number of downloads.

\section{Key Words}

Research tweets, Research downloads, Diabetes, Altmetric

\section{A Study of Factors Related to Readership of Scien- tific Articles}

\author{
Tony Xu, Shayan Khalili, Cynthia Deng \\ Earl Haig Secondary School \\ (EHSS 1)
}

\section{Abstract}

This report analysed the relationship between the number of Twitter and Mendeley views of scientific articles and the following factors: the GDP per capita of the country where the views came from, the title length, the publisher, the journal, and the subject of the article, in order to determine which factors affected how many views scientific articles accumulate. The purpose of this report was to help future researchers gain the most views possible on their published articles.

The data provided by Altmetric containing 550,000 json files, was extracted and displayed into several data frames, each one containing a different variable. The variables were then compared to views articles gained using a combination of bubble graphs, linear regression models, and scatter plots. From the results gathered, it could be seen that although the title length, the GDP per capita, and the subject of an article all affected the total views an article amassed, the journal and publisher had no notable effect on the views. In particular, title length within 41-100 characters had the most noticeable effect on the number of readers per article. Subjects, while showing that the more articles published about them brought in more total views, did not have a strong effect on the average number of viewers per article.

\section{Key Words}

Twitter, Mendeley, GDP per capita, Altmetrics

\section{Correlation Between Cancer Research Trends and Real World Data: An Analysis on Altmetric Data \\ Peter Chou, Kevin Hong, Chandler Lei, Haolin Zhang \\ Earl Haig Secondary School \\ (EHSS 2)}

\section{Abstract}

The field of cancer research is overall ambiguous to the general population and apart from medical news, not a lot is known of the proceedings. This study aims to provide some clarity towards cancer research, especially towards the correlations between research of different types of cancer. The amount of research papers pertaining to different types of cancers is compared against mortality and diagnosis rates to determine the amount of research attention towards a type of cancer in relation to its overall importance or danger level to the general population.

This is achieved through the use of many computational tools such as Python, R, and Microsoft Excel. Python is used to parse through the JSON files and extract the abstract and Altmetric score onto a single CSV file. $R$ is used to iterate through the rows of the CSV files and count the appearance of each type of cancer in the abstract. As well as this, $R$ creates the histograms describing Altmetric scores and file frequency. Microsoft Excel is used to provide further data analysis and find correlations between Altmetrics data and Canadian Cancer Society data.

The analysis from these tools revealed that breast cancer was the most researched cancer by a large margin with nearly 1,700 papers. Although there were a large number of cancer research papers, the Altmetric scores revealed that most of these papers did not gain significant attention.

By comparing these results to Canadian Cancer Society data, it was uncovered that Breast Cancer was receiving research attention that was not merited. There were four times more breast cancer research papers than the second most researched cancer, prostate cancer. This was despite the fact that breast cancer was fourth in mortality and third in new cases among all cancers. Inversely, lung cancer was underrepresented with only 401 research papers in spite of being the deadliest cancer in Canada.

\section{Key Words}

Cancer, Research, Treatment, Big data, Altmetrics 


\section{Evaluation of future trends of scientific research Charlie Sun, Kerry Li, Zhenyu Li \\ Earl Haig Secondary School EHSS 3}

\section{Abstract}

The rising trend of scientific researches have led more people to pay their attention towards scientific researches, but simply the word "scientific research" does not explain the whole nature of itself, like any other things in reality, it is divided into many realms. The various fields of scientific research have already been discussed by many scholarly articles and have been evaluated by previous census and researches. However, the ultimate question remains unanswered, namely, what is the most popular field of scientific research and which one will become the focus in the future.

Although the number of specific fields that can be derived is too vast to be counted, numerous major fields can be identified to categorize the various fields, such as astronomy, engineering, computer science, medicine, biology and chemistry. Several main factors are related to the popularity, such as the number of articles relating to respective fields, number of posts on social media and the number of views on professional sites.

A program was developed to analyze the relationship between the subjects for scientific research and the future trend of them based on the number of mentions for each field of research, scholarly articles and quotations about them. The program uses the data from Altmetric data, an authoritative data source. SAS is used to analyze the data and put the data on several graphs that represent the value for each factor. Finally, suggestions for future scientific researches can be summarized and inferred from the result of this research, which is aimed to provide enlightenment for future research directions.

\section{Key Words}

Big Data, Python, SAS, Research, Science

\section{The Future of Science: A Study on Interests Across the World}

\author{
Alex Bogdan, Yingning Gui, Henry Lin, Brennan Lu \\ Earl Haig Secondary School \\ EHSS 4
}

\section{Abstract}

This study was conducted to identify correlations between Altmetric data and its set of scientific topics. The scholarly articles published online were organized by distribution source, totaling the popularity of each article and its topic. In this case, topic refers to any scientific field of study and the data sets used were retrieved from Altmetric and Twitter sources. The purpose of this study is to help gain insight into where the future of science lies in subject matter as well as professional and public practice. as well as distinguish between what research the public favours to focus on in relation to privatized interests.

Publishing dates, Scopus subjects, and Altmetric scores were extracted from the Altmetric data. This data was compared with Twitter posters, helping differentiate the professional and public demographics that were interested in this field. Through the results, it was identified that the data showed that different cohorts - types of demographics - have different interests. Therefore, the future of science cannot be limited to single fields, rather an amalgamation of those popular among scholarly and public interests.

\section{Key Words}

Altmetric Score, Subject, Time, Big Data, Science, Cohorts

\section{Future of Science: Detecting Cancer}

Vic Li, Cecilia Shi, Michael Yang, Eva Zhang, Suzy Zhang, George Li

\section{Lowell International Academy \\ Lowell 1}

\section{Abstract}

Using Excel Data Analysis Tools and BigML Machine Learning platform, we tested correlation between biopsy data for breast cancer and created a model which helps to distinguish between benign and malignant tumors. Data set of oncology patients were used to analyze links between 10 indicators collected by biopsy non- can- 
cerous and cancerous tumours. Created model can be used as a future medical science tool and can be available to specially trained histology nurses in rural areas. Developed model that can be used to detect cancer on early stages is especially important in the view of the fact that detecting cancer at stage IV give patients of about $22 \%$ of survival rate 1 .

\section{Key Words}

Breast Cancer, Biopsy, BigML Modeling

\section{How Topics Affect Twitter Attention \\ Michal Fishkin, Jennifer Ou, Andrew Zhu \\ The Academy for Gifted Children - PACE (PACE 1)}

\section{Abstract}

The purpose of the investigation conducted was to discover trends in twitter popularity regarding different areas of science. This investigation can benefit areas of marketing such as targeted advertising, as well as demographic research in order to correctly test certain demographics and obtain research grants. Results included possible confirmation of our motive through principal component analysis, The data was compiled using RStudio and was narrowed down by subjects, Altmetric scores, and countries. The data was parsed through to find key words in the abstracts of articles. Principal Component Analysis was applied to a matrix of padded tweet dates, arranged by subject. These arranged dates were also plotted to visualize trends over time. From the data collected, the articles that were most tweeted about, between January 1st, 2016 to July 1st, 2016, worldwide were articles concerning physics. Out of all the articles, "death" was the keyword most popular in articles' abstracts. Disease-related words appeared far more often than the word "cure". The United States of America, Canada and Great Britain had the highest number of tweeters. Great Britain's population was mainly interested in articles regarding dentistry, while Canada and the United States of America had a higher tweet count in articles related to health science.

\section{Key Words}

Growth, Country, Subjects, Altmetric, score, Twitter

\section{An analysis of scientific fields by gender diversity through research paper metadata \\ Thomas Beckley, William Kwong, Danny Pechersky \\ The Academy for Gifted Children - PACE \\ (PACE 2)}

\section{Abstract}

The act of identifying interest in scholarly articles and research papers has long been difficult to quantify, let alone gather. In the past few years, altmetric data has allowed both researchers and the general public to access a wealth of information that was once difficult to collect. The ability to analyze public interest on scholarly articles and research papers allows for the writers themselves to identify general interest in a quantifiable manner. With such a large wealth of accessible information, general trends in regards to viewership can be extrapolated. Through social media, research articles are discussed, and their popularity is recorded into Altmetric's database. A major disparity plaguing most STEM fields currently is the lack of women in comparison to men in the STEM workforce. Thus, this study attempts to identify what scientific fields most interest each gender. To accomplish this, names and subjects were pulled from altmetric data. The names were input into a script to identify the gender of each name. The articles that a person has commented on has that person's name associated with its related scientific fields. The resulting data was combined and placed into various graphs to clearly visualize the disparity between different subjects and views by gender. The information was then analyzed. It was discovered that in terms of social media, more females viewed scholarly articles compared to men in most fields. However, it was found that papers relating to social sciences were viewed by more females compared to articles relating to material sciences, which garnered more male viewers.

\section{Key Words}

Gender, Twitter, Field, Subject, Interest 


\section{Exploring Government Involvement in Academia and its Effect on Social Media}

\author{
Natalie Nova, Michael Pavia, Andrew Schmittat \\ The Academy for Gifted Children - PACE \\ (PACE 3)
}

\section{Abstract}

Our team became very intrigued by the effect social media has on the world around us. This paper aims to see whether the government increasing their involvement in science was leading to more discussion of academia on twitter, or if it was the millennials in university who are bringing science and academia to social media. Using the Altmetric data sets, the Twitter API and the Geonames API we were able to construct a starting point for our project.

After getting the three countries who had the most tweets regarding scientific research papers and three countries who had the least we began to collect specific data for each country and compared them to find patterns. We observed that all of the top three countries had a very similar percentage of students in postsecondary education as well as a significant amount of federal funding. We also noticed that out of the bottom three countries they all had a drastically lower percentage rate of students. As for their federal funding Russia and India had a significantly lower budget but China had more federal funding than both United Kingdom and Germany. With this we can deduce that the relationship between how many tweets regarding scientific articles and university students is the most significant.

\section{Key Words}

Twitter, Geography, Locations, Government, Universities

\section{Effects of Climate Change on Canadian Forest Fires Leon Chen, Curtis Chong, Emily Huang, Nathan Lo Pierre Elliott Trudeau High School (PETHS 1)}

\section{Abstract}

This study aimed to determine the effects of climate change on forest fire trends in Canada by measuring correlations between weather conditions and the frequency and size of forest fires. Upon identifying the correlations, a model was created to understand fu- ture forest fire trends. The purpose of this study was to prevent the increasing trend of forest fires and devise solutions to reduce their damages.

The data obtained from the Canadian National Fire Database underwent a linear regression and a machine learning algorithm to respectively predict and correlate weather conditions with future forest fire trends. It was concluded that temperature and wind speed experienced a positive correlation with forest fire frequency and size and precipitation experienced a negative correlation.

To reduce the harmful effects of forest fires, cloud seeding can be used to create more precipitation and wind farms can be built to lower wind speed and attract lightning. However, more research and stricter policies directly targeting climate change are necessary for long term stability or decrease in forest fire trends.

\section{Key Words}

Forest Fire, Climate Change, Canada, Big Data

\section{The Role 3D Printers have in the Medical Field} Montgomery Gole, Sean Malins-Umansky, Patrick Prochazka, Tony Zhang

\section{Pickering College \\ (Pickering 1)}

\section{Abstract}

Gole, Malins-Umansky, Prochazka, and Zhang explore the idea around 3D printers and their future in modern medicine. They talk about how 3D printers have become a increasingly popular in the past years. They also talk about how scientists are becoming more and more interested in 3D printers and their applications in modern medicine. They give an example of a successful surgery where the doctor printed a spine and surgically implanted it into a 12-year- old bone cancer patient. They talk about how more operations like these will become possible in the future. They show data that looks at the increase of popularity over multiple different platforms of medical 3D printers. They show their code from python, explaining what it does and how they used it. They conclude that 3D printers are going to have a major role in modern medicine.

\section{Key Words}

Print, 3-Dimensional, Medicine, Tissue, Organs 


\section{An Analysis of Time and Engagement for Articles Relating to Oncology}

Joseph Train, David Roizenman, Seth Damiani, Ronny Rochwerg

\section{Tanenbaum CHAT Wallenberg Campus (Tanenbaum CHAT 1)}

\section{Abstract}

Oncology, the study of cancer, is a very broad and important field of science that many social media users do not know much about. The purpose of this paper is to determine which social networks have the most engagement about articles related to oncology. This will allow us to know optimal times and social networks to share research papers about Oncology that will have produce the highest engagement. By using the Python programming and Altmetric's API, we created several different graphs that display engagement with different types of cancer, and at what times they are being engaged with. The results we found prove that Twitter, Wikipedia, and Mendeley all have high engagement rates with all types of cancer. However, different kinds of cancer, such as breast cancer and prostate cancer, have different times throughout the year in which they receive more or less engagement on social media. The significance of these dates correlate with cancer awareness events around the world.

\section{Key Words}

Big Data, Oncology, Social Networks

\section{Statistical Analysis on Interdisciplinary Papers in 2016}

\author{
Lunjun Zhang, Justin Palombo, Sarah Costa \\ Villanova College \\ (Villanova 1)
}

\section{Abstract}

The goal of the project is to analyze altmetric data regarding published scientific papers in 2016, and to specifically determine which interdisciplinary fields are impactful. After taking some random samples, the program designed uses data clustering as well as data representation techniques to analyze the altmetric data. Trying to classify the papers into different levels of impact, k-means cluster- ing is applied in a creative way. With the focus on the interdisciplinary fields, three kinds of matrices are now calculated to illustrate the strength of the connection between every possible pairing combination: average altmetric score, percentage of published papers in this interdisciplinary field, and total altmetric score. Sorting based on the values obtained in the matrices and comparing three matrices can yield insightful results and help people understand the connections between different subjects better.

\section{Key Words}

Interdisciplinary Studies, Statistical Analysis, K-means Clustering, Altmetrics, Big Data

\section{Disease and the Steps to the Cure \\ Jamie Birker, Jesse Becker, Jessica Casalino \\ Villanova College}

(Villanova 2)

\section{Abstract}

An average of 56 million deaths occur worldwide each year and nearly $62 \%$ are caused by disease. Although research, awareness, and funding has increased significantly over the past century, it still might not be enough. After a small investigation it was revealed that the top three fatal diseases of Canadian citizens in 2015 were malignant neoplasms, heart diseases, and cerebrovascular diseases. A program which outputs key words, including ones related to health and medical sciences, was designed to quickly analyze the relevance of the aforementioned diseases. This program uses an input of data sets from 2016. Through analyzing the 2015 Top 100 articles and the 2016 data, we were able to infer the amount of research and funding that these topics receive, and its associated trends. Since these three diseases are leading causes of mortality rates, it is important that more research and funding goes towards these diseases, but more specifically the field of health and medical sciences.

\section{Key Words}

Disease, Big Data, Python, Malignant Neoplasms, Heart Disease, Cerebrovascular Disease 


\section{The Effect Computers Have on our Future Genera- tions}

\author{
Anya Filipas, Akera Otto, Lily Chen, Jenny Chen \\ Villanova College \\ (Villanova 3)
}

\section{Abstract}

With new advances of technology in recent years and upcoming research, it is certain that artificial intelligence will be making its way into the lives of many with accessible computers. As our younger generations are and will be greatly part of a technological world, it can be hypothesized that in the education systems, computer usage is prominent in learning, projects, and activities. This may affect the youth's mental and physical health and even their lifestyles, because being exposed to technology at a young age affects the way they do work and business grown-up. Overall, this is all about how, if computers are providing so much automatic service, aid, and information to children and teens, then they will probably be less productive, physically and mentally. As well, although the youth is constantly being given quick answers from the Internet, the overload of knowledge cannot help the fact that they will not be able to apply anything they learned to work. Given this information and the fact that we have an aging population, we hypothesize that words related to this aspect of society would be found among the results.

\section{Key Words}

Computer Usage, Learning, Information Overload

\section{Future demand and advancement in medical fields for best allocation of research funding \\ Valerie Hermanns, Daniel Grignano, Andrew Latobesi and \\ Mark Ho \\ Villanova College \\ (Villanova 4)}

\section{Abstract}

For a high school competition we were provided with access to altmetric data, and asked to predict the future of science. Based upon this, we shifted our focus to the medical sector, and to the correlation between future demand, and current research. Based on this focus, our goal was to predict which medical sectors will have the greatest need for research funding in the coming years. Our results will aid in the distribution of research funding in order to prepare for increased demand in medical fields that are currently not as prevalent. Using indicators of current research and development rates, as well as indicators of increasing demand in the future, we were able to design an algorithm and corresponding python programs which use three years worth of data to generate where research funds should be directed in the following two to three years. We also used lists of the top one hundred altmetric articles in 2014, 2015 and 2016 to calculate the average Altmetric Attention score, and number of papers in the top one hundred list for a variety of medical fields. Some interesting trends were found in these numbers, such as the major scientific influence viruses have the year after an outbreak.

\section{Key Words}

Funding, Demand, Research, Medical 